\title{
LITHOGRAPHICAL PATTERNING AND CARBONIZATION OF ELECTROSPUN SU-8 NANOFIBERS FOR A HIGH CAPACITY ELECTRODE
}

\author{
Gwan-Ha Kim", Gloria J. Kim² and Yong-Kyu "YK" Yoon" ${ }^{1 *}$
}

${ }^{1}$ Electrical Engineering, University at Buffalo, The State University of New York, Buffalo, NY 14260, USA

${ }^{2}$ Biomedical Engineering, University at Buffalo, The State University of New York, Buffalo, NY 14260, USA

\begin{abstract}
To fabricate large surface area conductive nanofibers for a high capacity electrode application in energy storage devices, we have successfully developed a three-step fabrication process: (1) generation of electrospun nanofibers with a photopatternable negative tone epoxy, SU-8, (2) lithographical patterning of the SU-8 nanofibers in a desired microscopic shape, and (3) subsequent thermal treatment for carbonization in an inert environment. Also, a multiple intermittent electrospinning scheme has been successfully established to stack the nanofibers as thick as $80 \mu \mathrm{m}$, demonstrating the possibility of three dimensional (3-D) nanofiber electrodes. This thickness can be further increased by repeating the scheme. We show the fiber diameter as a function of bias voltage and distance between the nozzle and the collector electrodes, the thickness of nanofiber stacks, lithographical patterning capability, and the electrical resistivity of the carbonized SU-8. This process will greatly impact research on high-density and fast-response battery and super capacitor development.
\end{abstract}

\section{INTRODUCTION}

Electrospinning provides a simple and a cost effective method for generating thin fibers from various materials that include polymers, composites, and ceramics [1]. The thin diameter of fibers provides a large surface area to volume ratio and superior mechanical performance that makes their extensive applications such as filtration for submicron or nanomaterials, separator, tissue scaffolding, drug delivery system, artificial organ and so on [2-5]. However, electrospun nanofibers are generally collected as two-dimensional membranes with randomly arranged structures and small bulk thickness, and also there are not much efficient ways available for micro scale patterning of such randomly grown electrospun nanofibers, which greatly limits their applicability for many future usages in biomedical, chemical, and nanotechnology. A few literatures have reported on the patterning of electrospun nanofibers requiring a dexterous fabrication technique [6-8]. Zhang and Chang have demonstrated that ultrafine electrospun nanofibers can be fabricated in three dimensional (3-D) fibrous tubes by a novel static method [9]. Li and colleagues have demonstrated that the nanofibers can be uniaxially aligned by introducing an insulating gap into the conductive collector [10]. However, these studies have limited their compatibility with other processes such as an accurate definition of 3-D microstructures using UV lithography patterning.

Meantime, the electrical characteristics of conductive polymers, such as polyaniline, polypyrrole, and polyethylene oxide, have attracted much interest recently [11-13]. However, the electrically conductive fiber shows relatively high resistivity. On the other hand, the carbonization of SU-8 microstructures is known to provide outstanding electrical, mechanical and chemical performance [14]. Very recently, research on electrospun SU-8 nanofibers and its carbonization have been reported by the authors [15]. We have further developed a simple method to prepare a bulk electrospun nanofibers and their patterning by using multiple times continuous growing and the UV lithography process, respectively. The advantages of this approach are twofold. It allows microscopic patterning and the process would be compatibility with the semiconductor process.

In this study, we demonstrate a thick stack of electrospun nanofibers using an intermittent approach, electrospun nanofiber patterning by using ultraviolet (UV) lithography, and the carbonization of electrospun nanofibers. Also, we report on the low resistivity carbon nanofibers. We investigate the controllability of the average nanofiber diameter, the thickness of patterned nanofiber structures, and an adequate resistivity of electrospun nanofibers.

\section{EXPERIMENTS DETAILS}

Random SU-8 nanofibers are fabricated using the electrospinning process under various conditions. SU-8 2025 (Microchem Inc.) is used as the working fluid. SU-8 2025 is diluted using cyclopentanone in a range of a concentration of $60.87 \%$ to $68.55 \%$ (by weight). All solutions are stored in room temperature and all experiments are conducted in room temperature in air.

The experimental setup used for the electrospinning process consists of an adjustable DC power supply (DEL HVPS MOD 603 $30 \mathrm{KV}$ POS, Spellman High Voltage Electronic Corp., USA) capable of generating DC voltage in a range of $0-30 \mathrm{kV}$, a syringe pump (NE-1000, New Era Pump Systems, Inc., USA) on which a 5 $\mathrm{ml}$ syringe is connected with a stainless steel needle having an inner diameter of $0.2 \mathrm{~mm}$. The working distance between the needle and the collector is in a range of $7.5-25 \mathrm{~cm}$. Positive voltages applied to SU-8 solutions are in a range of $12.5-17.5 \mathrm{kV}$. The solution flow rates are controlled by a syringe pump and the pumping rate is 0.02 $\mathrm{ml} / \mathrm{min}$. The electrospun nanofibers have diameters ranging between $340 \mathrm{~nm}$ and 3.3um, depending upon different electrospinning conditions. The diameter of SU-8 electrospun nanofibers is measured by a field emission scanning electron microscopy (FE-SEM) system (SU-70, Hitachi, Japan). A UV exposure system (LS30, OAI, Inc) has been used for the patterning of electrospun nanofibers.

A thick stack of electrospun SU-8 nanofibers are deposited on a Si substrate (Figure 1a). Ultraviolet (UV) exposure (i-line, $\lambda=365 \mathrm{~nm}$ ) is applied for patterning the nanofibers, followed by post exposure bake (Figure 1b). After the electrospun nanofibers are
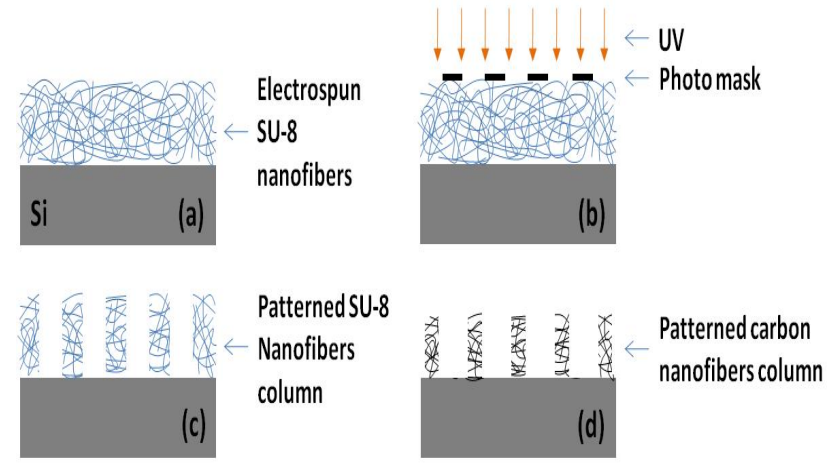

Figure 1. Schematic of the fabrication process: (a) preparation of electrospun SU-8 nanofibers, (b) lithographic patterning of fibers, (c) development of $U V$ exposed fibers, and (d) formation of patterned carbon nanofiber columns by pyrolysis. 
developed (Figure 1c), the substrate undergoes pyrolysis (Figure 1d). Electrospun SU-8 nanofibers are converted into the carbon nanofiber by pyrolysis $[14,15]$.

The resistivities of carbonized SU-8 thin films are measured by using a four-point probe head (C4S, Cascade Microtech, Inc., USA), a current source (HP 6177C, HP, USA), a current meter (194A, Keithley Instruments Inc., USA), and a voltage meter (195A, Keithley Instruments Inc., USA), at room temperature. And the Auger electron microscopy (AES) (Microlab 310-D, Thermo VG Scientific, USA) analysis is conducted to verify change in the composition of electrospun nanofibers after pyrolysis.

\section{RESULTS AND DISCUSSION}

SU-8 Electrospun nanofibers are fabricated by the electrospinning process under various conditions. One of the most important parameters among the controlled variables is the effect of the applied voltage and working distance between the needle and the collector. Figure 2 shows the variation of an average electrospun fiber diameter as a function of distance between the needle and the collector at various applied voltages. The average diameter of electrospun nanofibers becomes smaller as the travel distance increases due to: (i) more solvent evaporation, and (ii) continuous stretching due to electrostatic force $[16,17]$. As the working distance increases, the average diameter of electrospun fibers decreases. It has been found that a minimum distance is required to allow the fibers to have sufficient time to remove solvent before reaching the collector [18].

Meanwhile, the average diameter of the electrospun fibers increases when a higher voltage is applied. In case of SU-8, the higher voltages yield the larger fiber diameters. In electrospinning, the charge transport under the applied electric field is a main mechanism for electrospun fiber deposition, which is attributed to | the mass flow of_SU-8 from the needle tip. Deitzel and colleagues have reported that an increase in applied voltage causes a change in the shape of the jet initiating point, and hence the structure and morphology of fibers [19]. In case of SU-8, the diameter of electrospun fibers largely varies depending on the applied voltage.

The relationship between the SU-8 concentrations and the diameter of electrospun fibers has been studied. Solution

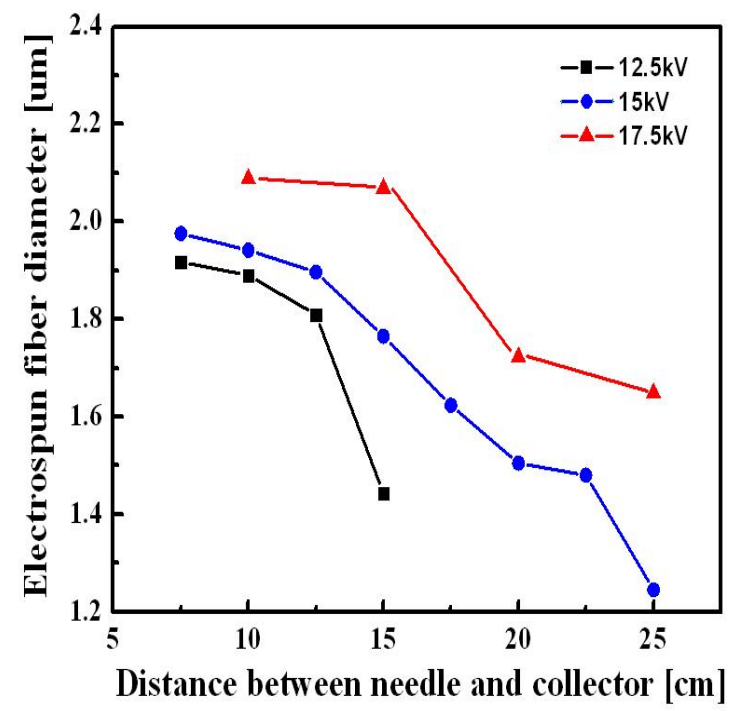

Figure 2. Variation in the diameter of electrospun fibers as a function of distance between the needle and the collector at various applied voltages.

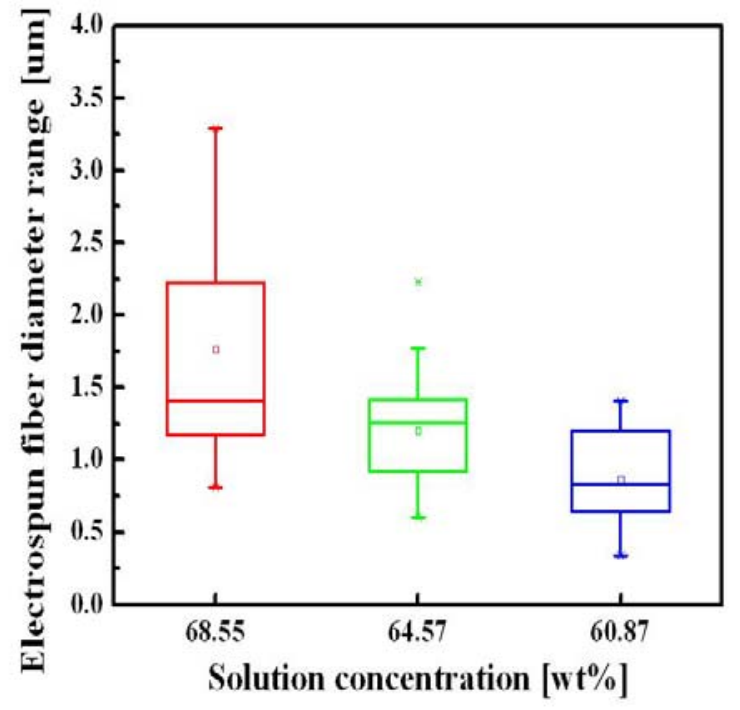

Figure 3. Variation in electrospun fiber diameter as a function of SU-8 solution concentration.

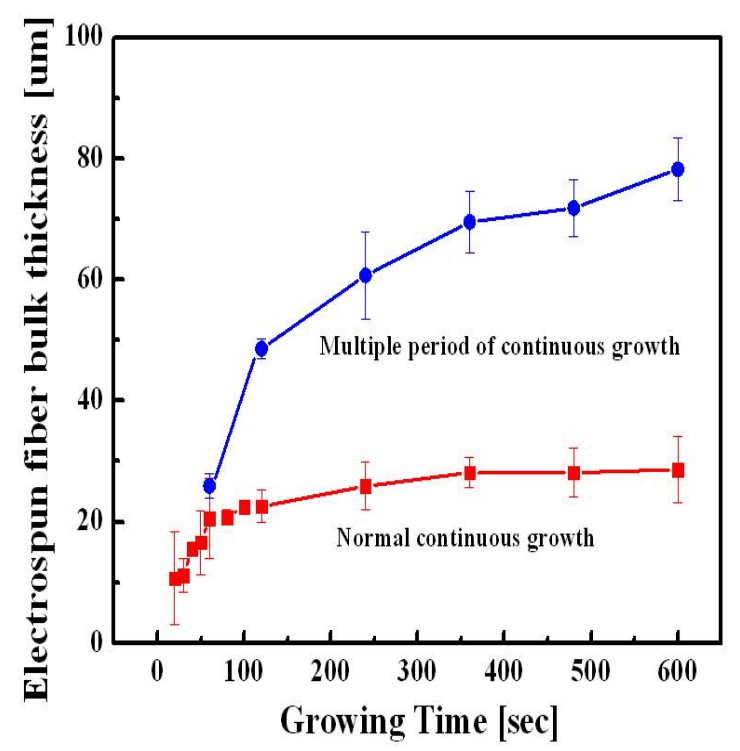

Figure 4. Effect of multiple periods of continuous growth and normal continuous growth on nano fiber stacking.

concentration along with viscosity and surface tension affects the condition of the electrospun fiber formation [20]. Figure 3 shows a distribution of fiber diameters obtained from SU-8 electrospinning with three different concentrations of $68.55,64.57$, and $60.87 \mathrm{wt} \%$ and all other variables maintained constant. A decrease in solution concentration results in fibers with smaller diameters. In this case, decreasing the concentration of a SU-8 solution can also affect its surface tension. The effects of solution properties on fiber diameter have previously been discussed for other polymeric systems [17, 19]. As seen with other polymers, SU-8 has a linear relationship between solution concentration and resulting fiber diameter. The increase in viscosity that comes with concentration increase causes this effect. These relationships indicate that solution concentration plays an important role in determining the fiber diameter.

Although we can obtain a uniform and appropriate thickness of the electrospun fiber, we need a thicker bulk electrospun fiber stack 
for some applications like an energy storage device, electronic device, and bio chemical applications. For such applications, high fiber packing density and high porosity may be desirable. We demonstrate an easier approach. Figure 4 shows two electrospinning conditions: multiple periods of continuous deposition (growth) and normal continuous deposition (growth) of electrospun fibers. These plots can be divided into two sections. When SU-8 fiber growing time is less than $60 \mathrm{sec}$, the growth rate of SU-8 electrospun fibers increases linearly. But, growth rate is saturated after $60 \mathrm{sec}$ as shown in the normal continuous growth plot. It is attributed to that deposited electrospun fibers on the collector are positively charged due to the slow discharge time of the positive ions through the none conducting nanofibers. Accumulated positive ions in the electrospun fibers are repelling the subsequent nanofibers and preventing further growing. This charge repelling phenomenon has been much relieved by using a multiple periods of continuous growth approach where the electrospinning process has been performed for $30 \mathrm{sec}$ followed by a rest period of $30 \mathrm{sec}$ before the next electrospinning step begins. Charge in the electrospun fibers is slowly discharged during the rest time. And the repelling force has been significantly reduced and we have achieved a thick stack of electrospun fiber upto $80 \mathrm{um}$ after twelve $30 \mathrm{sec}$ cycles.

For micro/nanometer scale integrated devices, accurate definition of electrospun fibers using ultraviolet (UV) lithography will be very useful. Although patterned electrodes or micro-contact printing can allow nanofiber patterning to some extent [19], it is quite limited from the viewpoint of the patternable thickness and the patternability in arbitrary shape. A new approach applying UV lithography directly on electrospun nanofibers is proposed and detailed. This approach does not require an additional process like a reactive ion etching process after lithography or micro molding. Figure 5 show SEM images of (a) a line with a width of $120 \mu \mathrm{m}$ and (b) a circle with a diameter of $100 \mu \mathrm{m}$. While some edges look rough because of the edge of some nanofibers, the overall shape is well defined by the original photo mask geometry with a good fidelity.

In addition, these patterned nanofibers have been further processed using pyrolysis, converting the patterned electrospun fibers into carbon nanofibers, resulting in chemically and

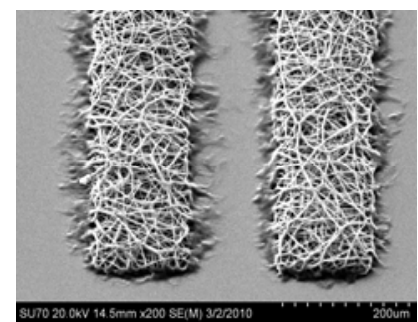

(a)

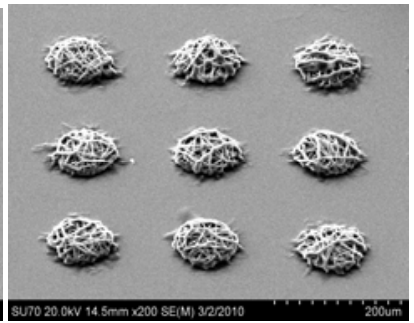

(b)
Figure 5. SEM images of patterned SU-8 electrospun fibers: (a) $120 \mu \mathrm{m}$ wide lines and (b) $100 \mu \mathrm{m}$ diameter circles.

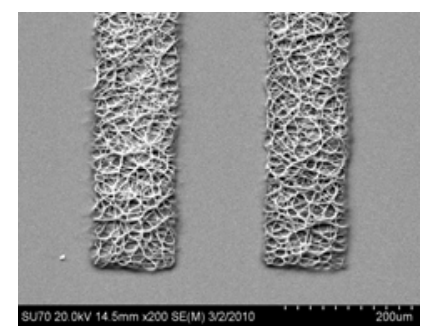

(a)

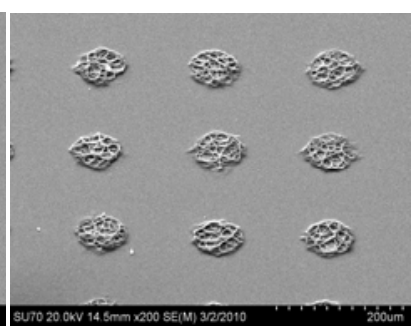

(b)
Figure 6. SEM images of pyrolyzed SU-8 electrospun fiber patterns: (a) $120 \mu \mathrm{m}$ wide lines and (b) $100 \mu \mathrm{m}$ diameter circles.

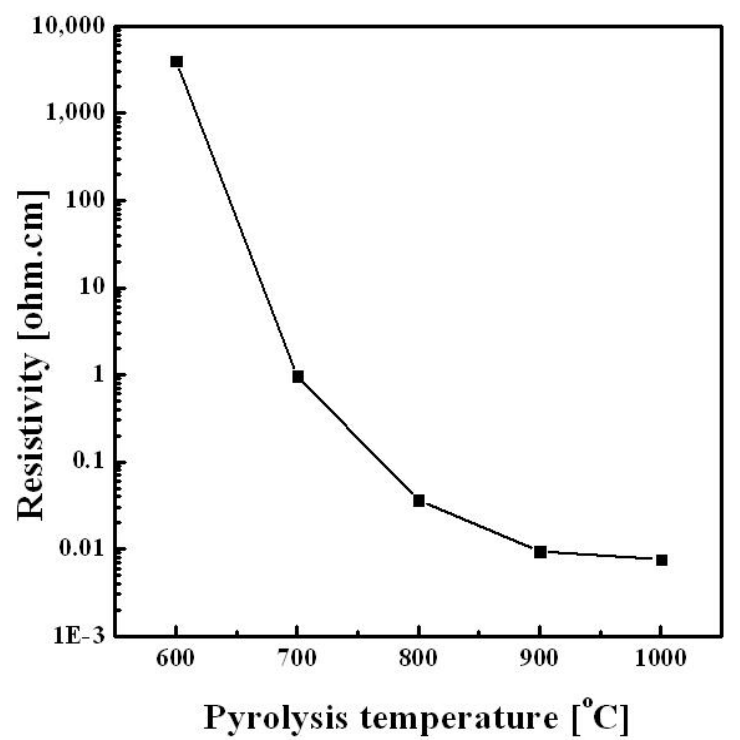

Figure 7. Resistivity of pyrolyzed SU-8 as a function of pyrolysis temperature.

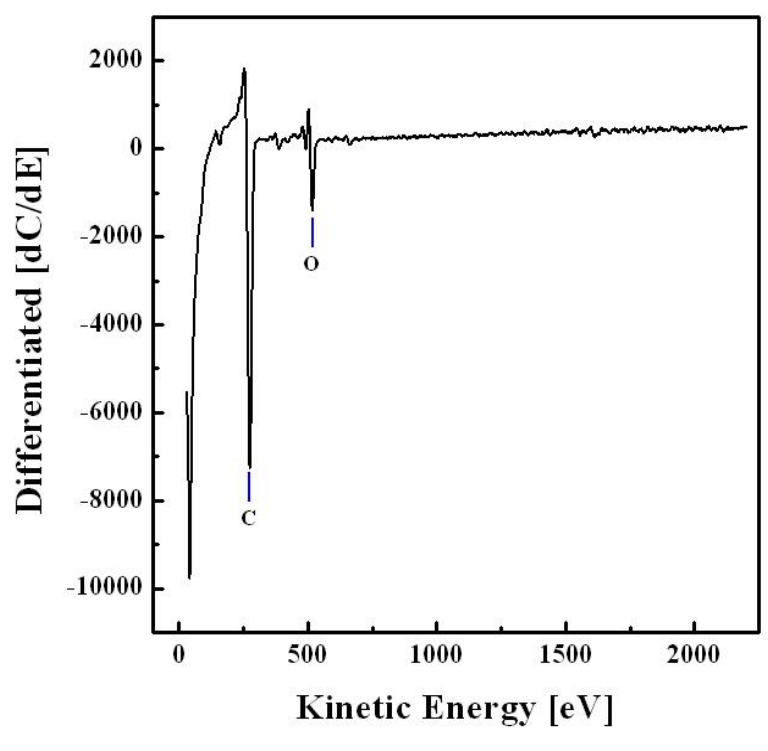

Figure 8. AES analysis of pyrolyzed SU-8 electrospun fiber.

mechanically stable, low cost, high surface area electrodes. Carbon nanofibers are finding enormous applications in unconventional energy sources and storage cells due to their enhanced conductivity and high aspect ratio. Carbon nanofiber reinforced composites offer increased stiffness, high strength and low electrical resistivity [21]. Figure 6 shows SEM images of patterned electrospun fibers of (a) a line with a line width of $120 \mu \mathrm{m}$ and (b) a circle with a diameter of $100 \mu \mathrm{m}$, obtained after pyrolysis in a nitrogen purged quartz tube furnace.

A thin SU-8 film is prepared for resistivity measurement after the pyrolysis by using four-point probe system. Figure 7 shows the resistivity of the pyrolyzed carbon film at different pyrolysis temperatures. The resistivity decreases as the pyrolysis temperature increases. The decrease in resistivity with an increase in temperature is attributed to the degree of graphitization [22]. The higher 
temperature of pyrolysis, the higher the extent of graphitization, and thus the lower resistivity.

The AES analysis has been conducted to verify change in composition of electrospun fibers after the pyrolysis. The direct analysis of the surface composition of carbon film can give us certain information about it. Figure 8 shows an AES surface scan result on the carbon film after the pyrolysis of a thin SU-8 film. From the AES surface scan result, it is expected that all polymer components in SU-8 has been changed into carbon. The existence of the oxygen element may be due to the exposure of the sample in air before and during AES analysis.

\section{CONCLUSION}

We have highlighted studies that examine the effect of each parameter in the SU-8 (a photopatternable negative tone epoxy) electrospinning process. Electrospun SU-8 nanofibers have been successfully obtained. The diameter of the fiber is affected by the solution concentration, electric field and the distance between the needle tip and the collector. A multiple periods of continuous growth method enables thick bulk electrospun fiber deposition. After twelve 30 second cycles in alternating electrospinning and pose steps, a stack as thick as $80 \mu \mathrm{m}$ has been successfully obtained. The achievable thickness is not limited to the thickness though. Furthermore, a conventional UV lithography process has been used to pattern the electrospun fibers. This method greatly facilitates the micropatterning of nanofibers and must be very versatile and useful for micro/nano fiber integrated devices requiring accurate pattern size and shape. The patterned electrospun SU-8 nanofibers have been further pyrolized, turning into the patterned carbon nanofibers, which are considered as a good candidate of a high capacity electrode for high density energy storage devices such microbatteries and capacitors.

\section{ACKNOWLEDGEMENT}

This work is supported by the National Science Foundation (CAREER:ECCS 0748153 and CMMI 0826434) and the Korea Research Foundation Grant funded by the Korean Government (KRF-2008-357-D00104). Also, the authors would like to thank Dr. Seong-Hyok Kim and Dr. Mark G. Allen in the Georgia Institute of Technology for valuable discussion and some dielectric material deposition.

\section{REFERENCES}

[1] D. Li and Y. Xia, "Electrospinning of nanofibers: Reinventing the Wheel", Adv. Mater., 16, 1151 (2004).

[2] J.R. Kim, S.W. Choi, S.M. Jo, W.S. Lee, and B.C. Kim, "Characterization and Properties of PVdF-HFP-Based Fibrous Polymer Electrolyte Membrane Prepared by Electrospinning", J. Electrochem. Soc., 152, A295 (2005).

[3] W.-J. Li, C.T. Laurencin, E.J. Caterson, R.S. Tuan, and F.K. Ko, "Electrospun nanofibrous structure: A novel scaffold for tissue engineering", Journal of Biomedical Materials Research Part A, 60, 613 (2002).

[4] J.R. Venugopal, S. Low, A.T. Choon, A.B. Kumar, and S. Ramakrishna, "Nanobioengineered Electrospun Composite Nanofibers and Osteoblasts for Bone Regeneration", Artificial Organs, 32, 388 (2008).

[5] N.M. Neves, R. Campos, A. Pedro, J. Cunha, F. Macedo, and R.L. Reis, "Patterning of polymer nanofiber meches by electrospinning for biomedical applications", International Journal of Nanomedicine, 2, 433 (2007).

[6] E.P.S. Tan, S.Y. Ng, and C.T. Lim, "Nano tensile testing of a single ultrafine polymeric nanofiber", Biomaterials, 26, 1453
(2005).

[7] R. Inai, M. Kotaki, and S. Ramakrishna, "Structure and Properties of Electrospun PLLA Single Nanofibres", Nanotechnology, 16, 208 (2005).

[8] Y. Ishii, H. Sakai, and H. Murata, "Fabrication of a submicron patterned electrode using an electrospun single fiber as a shadow-mask", Thin Solid Films, 518, 647 (2009).

[9] D. Zhang and J. Chang, "Electrospinning of Three-Dimensional Nanofibrous Tubes with Controllable Architectures", Nano Letters, 8, 3283 (2008).

[10] D. Li, G. Ouyang, J.T. McCann, and Y. Xia, "Collecting Electrospun Nanofibers with Patterned Electrodes", Nano Letters, 5, 913 (2005).

[11] I.D. Norris, M.M. Shaker, F.K. Ko, and A.G. MacDiarmid, "Electrostatic Fabrication of Ultrafine Conducting Fibers: Polyaniline/Polyethylene Oxide Blends," Synth. Met., 114, 109 (2000).

[12] I.S. Chronakis, S. Grapenson, and A. Jakob, "Conductive polypyrrole nanofibers via electrospinning: Electrical and morphological properties", Polymer, 47, 1597 (2006).

[13] J.W. Lu, Y.L. Zhu, Z.X. Guo, P. Hu and J. Yu, "Electrospinning of sodium alginate with poly(ethylene oxide)", Polymer, 47, 8026 (2006).

[14] C. Wang, G. Jia, L.H. Taherabadi, and M.J. Madou, "A Novel Method for the Fabrication of High-Aspect Ratio C-MEMS Structures", Journal of Microelectromechanical Systems, 14, 348 (2005).

[15] G.H. Kim, E. Kozarsky, H.S. Jee, K.T. Kim. J. Kim, E. Takeuchi, and Y.K. Yoon, "Carbon Nanotube Embedded Three Dimensional (3-D) Carbon Microelectrodes for Rechargeable Microbatteries", AVS 56th International Symposium \& Exhibition, 11/8-13/09, San Jose, CA (2009).

[16] J. Doshi and D.H. Reneker, "Electrospinning Process and Applications of Electrospun Fibers”, J. Electrostatics, 35, 151 (1995).

[17] M.M. Demir, I. Yilgor, E. Yilgor, and B. Erman, "Electrospinning of polyurethane fibers", Polymers, 43, 3303 (2002).

[18] X.Y. Geng, O.H. Kwon, and J.H. Jang, "Electrospinning of chitosan dissolved in concentrated acetic acid solution", Biomaterials, 26, 5427 (2005).

[19] J. Shi, L. Wang, and Y. Chen, "Microcontact Printing and Lithographic Patterning of Electrospun Nanofibers", Langmuir letter, 25, 6015 (2009).

[20] J.M. Deitzel, J. Kleinmeyer, D. Harris, N.C. Beck Tan, "The Effect of Processing Variables on the Morphology of Electrospun Nanofibers and Textiles", Polymer, 42, 261 (2001).

[21] T. Subbiah, G.S. Bhat, R.W. Tock, S. Parameswaran, S.S. Ramkumar, "Electrospinning of Nanofibers", Journal of Applied Polymer Science, 96, 557 (2005).

[22] A. Singh, J. Jayaram, M.J. Madou, and S. Akbar, "Pyrolysis of negative photoresists to fabricate carbon structures for microelectromechanical systems and electrochemical applications", Journal of the electrochemical Society, 149, E78 (2002).

\section{CONTACT}

Gwan-Ha Kim gwanhaki@buffalo.edu ,

*Yong-Kyu Yoon, tel: +1-716-645-1029; ykyoon@,buffalo.edu 